\title{
Combination of vascular endothelial growth factor-loaded microspheres and hyperbaric oxygen on random skin flap survival in rats
}

\author{
XUE-GUAN XIE $^{1 *}$, MEI ZHANG $^{2 *}$, YAN-KUN DAI $^{1}$, MING-SHENG DING $^{1}$ and SHENG-DONG MENG ${ }^{1}$ \\ ${ }^{1}$ Department of Orthopedics, Huai'an Hospital Affiliated of Xuzhou Medical College and Huai'an Second People's Hospital, \\ Huai'an, Jiangsu 223002; ${ }^{2}$ The Second Department of Nursing, Jiangsu Vocational College of Nursing, \\ Huai'an, Jiangsu 223300, P.R. China
}

Received September 1, 2014; Accepted June 16, 2015

DOI: $10.3892 / \mathrm{etm} .2015 .2620$

\begin{abstract}
The present study aimed to investigate the role of hyperbaric oxygen (HBO) and treatment with vascular endothelial growth factor (VEGF)-loaded microspheres, and HBO plus VEGF on the survival of random skin flaps in rats. The modified McFarlane flap model was established in 40 rats and evaluated within four groups: VEGF $(n=10), \mathrm{HBO}$ $(n=10)$, HBO plus VEGF $(n=10)$ and controls $(n=10)$. Seven days following treatment, the necrotic area of the flap was measured. The specimens were stained with hematoxylin and eosin for histological analysis. Immunohistochemical staining was used to analyze the positive expression levels of VEGF. The percentages of necrosis of the skin flaps in all groups were: $49.66 \pm 2.64 \%$ in controls, $26.85 \pm 1.77 \%$ in VEGF, $28.27 \pm 2.21 \%$ in $\mathrm{HBO}$ and $10.44 \pm 2.48 \%$ in the combination group. Histological analysis demonstrated angiogenesis with mean vessel density per $\mathrm{mm}^{2}$ in the groups were: $16.68 \pm 2.69$ in controls, 22.96 \pm 3.29 in VEGF, 24.74 \pm 3.19 in $\mathrm{HBO}$ and $34.81 \pm 3.93$ in the combination group. The expression of VEGF of the controls, VEGF, HBO and the combination group were $28.33 \pm 4.98,52.54 \pm 4.55,49.32 \pm 4.62$ and $78.97 \pm 4.90$ integral absorbance, respectively. For all measurements, the combination group showed greater improvement in random skin flap survival than others $(\mathrm{P}<0.05)$. No significant difference was detected between the VEGF and HBO group. The control group exhibited lower survival rates compared with the other groups $(\mathrm{P}<0.05)$. Combination of VEGF and HBO improved
\end{abstract}

Correspondence to: Dr Xue-Guan Xie, Department of Orthopedics, Huai'an Hospital Affiliated of Xuzhou Medical College and Huai'an Second People's Hospital, 62 Huaihai South Road, Huai'an, Jiangsu 223002, P.R. China

E-mail: xueguanxie@163.com

*Contributed equally

Key words: vascular endothelial growth factor, hyperbaric oxygen, random skin flap survival, necrosis, angiogenesis random skin flap survival compared with the effect of VEGF or HBO alone, suggesting these two agents exhibited a synergistic effect.

\section{Introduction}

Random pattern skin flaps are common for wound repair and reconstruction. Skin flap grafting is widely used in plastic surgery to repair defects resulting from trauma, congenital disease and tumor excision. In clinical practice, flap necrosis, which may be caused by lack of adequate nutrient blood supply, is a serious problem $(1,2)$. Therefore, exploring possible targets for therapeutic intervention to reduce flap necrosis and increase ischemic tissue survival is important.

A number of studies have been conducted to improve random pattern skin flap survival. For example, angiogenic growth factors, such as vascular endothelial cell growth factor (VEGF) improved skin flap survival by enhancing the revascularization of ischemic tissues $(3,4)$. VEGF is a specific mitogen for endothelial cells and it stimulates vasculogenesis and angiogenesis. It can promote vascular endothelial cell proliferation and migration, promote angiogenesis, extend the life of the vascular endothelial cells and increase vascular permeability (5). In addition to VEGF, it has also been reported that hyperbaric oxygen (HBO) could increase the tolerance of tissues to ischemia and thus improve the survival probability $(2,6,7)$. HBO could protect the microcirculation through interfering with the deleterious activity of neutrophils (8). The effects of VEGF and HBO in improving the survival of a random skin flap are already known. However, information regarding the effect of the combination of VEGF and $\mathrm{HBO}$ is unclear.

Considering the fact that $\mathrm{HBO}$ could increase the expression of VEGF (9) and both could promote angiogenesis $(3,4,10)$, it was hypothesized that dual treatment of VEGF-loaded microspheres and HBO would result in improved random pattern skin flap survival. To test this hypothesis, the effects of HBO, VEGF loaded microspheres, and $\mathrm{HBO}$ plus VEGF on the survival of random skin flaps in rats were analyzed. 


\section{Materials and methods}

Ethics statement. The study was approved by the ethics committee of Huai'an Hospital Affiliated of Xuzhou Medical College and Huai'an Second People's Hospital (Huai'an, China). All procedures strictly followed the recommendations in the Guide for the Care and Use of Laboratory Animals of the National Institutes of Health.

Animals and materials. Forty Male Sprague-Dawley (SD) rats (weight, 200-250 g; age, 2-3 moths) were obtained from the laboratory animal center of Xuzhou Medical College (Xuzhou, China). VEGF was purchased from Boyun Biotech Co., Ltd. (Shanghai, China). Mice anti-rat VEGF antibodies (sc-7269) were purchased from Santa Cruz Biotechnology Inc. (Santa Cruz, CA, USA). The goat anti-mouse secondary antibody and goat-anti-rabbit secondary antibody (R125) were purchased from Boyun Biotech Co., Ltd. (Shanghai, China). Antigen retrieval was achieved by microwaving (10 $\mathrm{min}$, $700 \mathrm{~W}$ ) in citrate buffer solution at pH 6 (BY02072; Boyun Liyuan Biotechnology Co., Ltd., Shanghai, China). HBO chamber was designed by the Wuhan Ship Development and Design Institute (Wuhan, China).

Preparation of VEGF-loaded microspheres. For microsphere preparation, $1.2 \mathrm{~g}$ gelatin (Type B, 225 Bloom Sigma-Aldrich, St. Louis, MO, USA) was firstly dissolved in $4 \mathrm{ml}$ double-distilled water in a water bath at $55^{\circ} \mathrm{C}$. The gelatin solution was added dropwise to $30 \mathrm{ml}$ liquid paraffin (containing $10 \mathrm{~g} / 1$ Span-80; Sigma-Aldrich), which was preheated to $55^{\circ} \mathrm{C}$, to form a water in oil emulsion by stirring (450 rpm for $10 \mathrm{~min}$ ). Subsequently, the emulsion was chilled at $4^{\circ} \mathrm{C}$ and gelatin microspheres were formed in the aqueous phase, then $0.1 \mathrm{ml}$ glutaraldehyde $(250 \mathrm{~g} / \mathrm{l})$ precooled at $4^{\circ} \mathrm{C}$ was added. The resulting microspheres were filtered and rinsed in acetone, isopropyl alcohol and diethyl ether to remove the remaining oil on their surfaces. Finally, the rinsed gelatin microspheres were dried in a vacuum oven. Encapsulation of VEGF was then achieved by diffusional loading. Briefly, phosphate-buffered saline (PBS) solution containing bovine serum albumin $(0.1 \% \mathrm{w} / \mathrm{v}, 200 \mu \mathrm{l})$ and VEGF (1 mg/1 ml) added to sterilized microspheres (20 mg) and left for $24 \mathrm{~h}$. Subsequently, the loaded microparticles were washed, centrifuged at 10,000 x g and sterilized. To investigate the VEGF release, $10 \mathrm{mg}$ microspheres were placed in a tube containing $100 \mathrm{ml} \mathrm{PBS}$ at $37^{\circ} \mathrm{C}$. The tube was continuously shaken using a JJ21 electronic mixer (135 rpm; Changzhou Guohua Electric Appliance Co., Ltd., Changzhou, China) and at particular time intervals (every $24 \mathrm{~h}$ for 7 days) the samples were centrifuged at $10,000 \mathrm{x} \mathrm{g}$, supernatant aliquots were collected and replaced with an equal volume of PBS. The concentration of VEGF in the supernatant was measured with commercial enzyme-linked immunosorbent assay kits (R\&D Systems, Inc., Minneapolis, $\mathrm{MN}, \mathrm{USA})$. The release tests were conducted in triplicate.

Flap model and experimental design. Under sterile conditions, all rats were anesthetized with $10 \%$ chloral hydrate ( $3 \mathrm{ml} / \mathrm{kg}$; Sigma-Aldrich) by intraperitoneal injection. The dorsal region was shaved and disinfected with povidone iodine (PI) solution (Sigma-Aldrich). Random dorsal skin flaps were elevated using the Improved McFarlane flap method as described previously $(11,12)$. A rectangular area $\left(3 \times 9 \mathrm{~cm}^{2}\right)$ was outlined on the back of the rats and the sacral arteries were systematically sectioned. The flap was completely separated from the underlying fascia and then immediately sutured back to the donor bed using 4-0 sutures (Ailee Co., Ltd., Busan, South Korea). Regions around the incision were disinfected with iodine PI solution and smeared with aureomycin ointment (Sigma-Aldrich). For subsequent analysis, the flap area was divided into three zones of equal size: The proximal area I the intermediate area II and the distal area III (13). The 40 rats were randomly divided into four groups. The VEGF group received $3 \mathrm{ml}$ microsphere PBS solution $(2 \mu \mathrm{g} / \mathrm{ml})$ after the elevation of skin flap. The microspheres were delivered in two sites, one site was $3 \mathrm{~cm}$ distant from the distal end and the other was $6 \mathrm{~cm}$ distant from the distal end, and each site received a $1.5-\mathrm{ml}$ injection. For the HBO group, the rats were firstly injected with $3 \mathrm{ml}$ saline. After $30 \mathrm{~min}$ the rats were then moved to the HBO chamber (2.5 standard atmospheric pressure, oxygen concentration over $98 \%$ ) for $30 \mathrm{~min}$. After $8 \mathrm{~h}$, the rats were moved into the $\mathrm{HBO}$ chamber again for $30 \mathrm{~min}$. The dual treatment group was firstly injected with $3 \mathrm{ml}$ VEGF microsphere solution. After $30 \mathrm{~min}$, the rats were moved to the $\mathrm{HBO}$ chamber for $30 \mathrm{~min}$ (twice per day at $8 \mathrm{~h}$ intervals). The control group only received $3 \mathrm{ml}$ saline daily. All treatments were performed consecutively every $24 \mathrm{~h}$ for 7 days. All rats were housed individually and fed standard rat chow and water ad libitum. Each rat was given a neck collar to prevent self-mutilation (14).

General observation and percentage of necrosis. On the seventh day, the flap area was photographed using a BX51 optical microscope (Olympus Corporation, Tokyo, Japan) and compared with the record on the first day. The necrotic area was defined according to the dark color and eschar formation. The photos were captured by the software Image-Pro Plus 6.0 (Media Cybernetics, Inc., Rockville, MD, USA). For each group, the mean and standard deviation of the necrotic area percentage were calculated.

Histology. After the rats were sacrificed with an overdose of chloral hydrate, flap tissues from all zones were conventionally dehydrated, embedded, sectioned and stained (hematoxylin and eosin). Tissue condition, such as the thickness of granulation tissue, tissue edema, necrosis, hyperplasia of capillaries, blood vessels and inflammatory cell infiltration of each layer was observed under the BX51 light microscope (magnification, x100). In addition, the microvessel number per unit area (per $\mathrm{mm}^{2}$ ) was also determined as an indicator of the microvascular density (MVD) (15).

Immunohistochemistry for VEGF expression. Immunohistochemical staining was conducted for VEGF using the streptavidin-peroxidase method (16). Positive VEGF expression in each flap was observed under an inverted microscope and analyzed the image through the software Image-Pro Plus 6.0. The integral absorbance (IA) value was detected as an indicator of VEGF expression. 
A

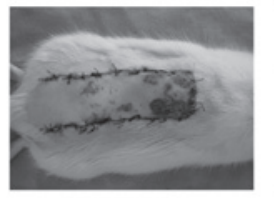

Combined

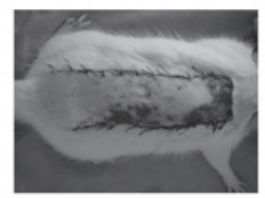

VEGF

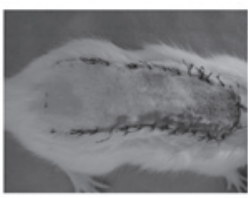

Hyperbaric oxygen

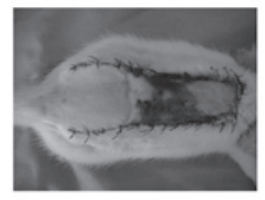

Control

B

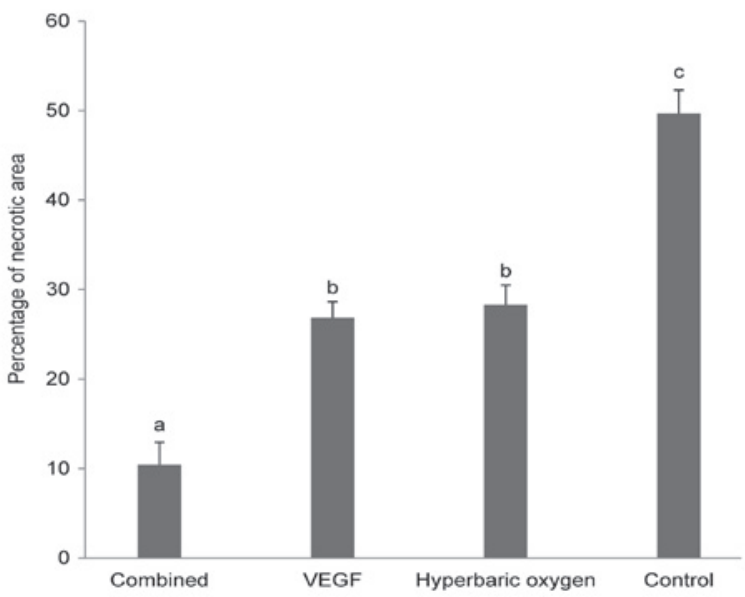

Figure 1. Comparison of the necrotic flap area between the four groups. The histogram with bars indicate the mean \pm standard deviation. Different letters indicate significant differences at the $\mathrm{P}<0.05$ level. VEGF, vascular endothelial growth factor.

A

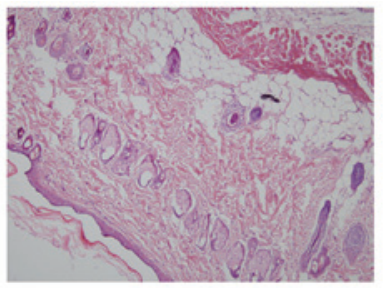

Combined

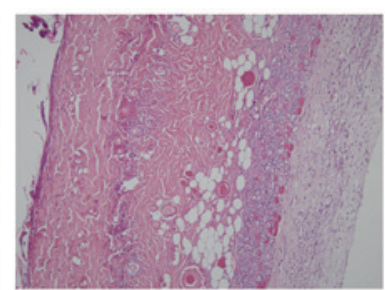

VEGF

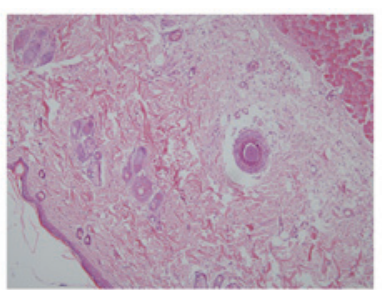

Hyperbaric oxygen

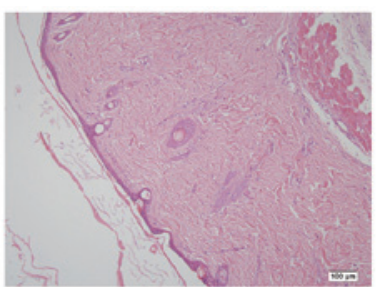

Control

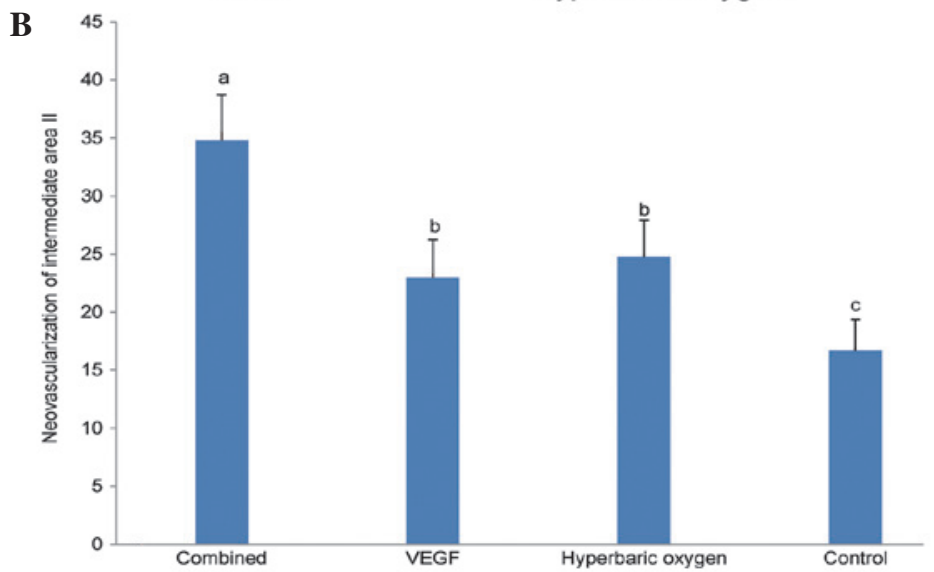

Figure 2. Comparison of angiogenesis in the intermediate area II between the four groups. (A) Representative fields from samples of the various groups (stain, hematoxylin and eosin; magnification, x100). (B) Histogram with bars indicating the mean \pm standard deviation. Different letters indicate significant differences at the $\mathrm{P}<0.05$ level. VEGF, vascular endothelial growth factor.

Statistical analysis. All results are expressed as the mean \pm standard deviation for continuous variables. The data were analyzed using SPSS software, version 17.0 (SPSS Inc., Chicago, IL, USA). P $<0.05$ was considered to indicate a statistically significant difference. Statistical analysis consisted of a comparison of means of each group by using analysis of variance. Tukey's multiple comparison test was applied when appropriate.

\section{Results}

Microsphere characterization and VEGF release. The obtained microspheres were spherical, smooth and non-porous. Over $85 \%$ microspheres had a diameter of $10-28 \mu \mathrm{m}$. Loading VEGF did not induce any change in the morphology or size range. The ability of the microspheres to provide a sustained delivery of VEGF was assessed in preliminary experiments. 


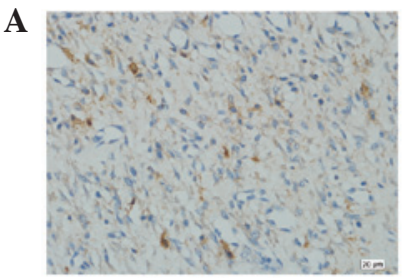

Combined

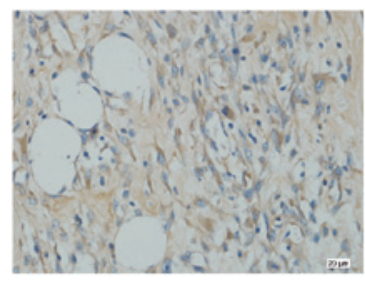

VEGF

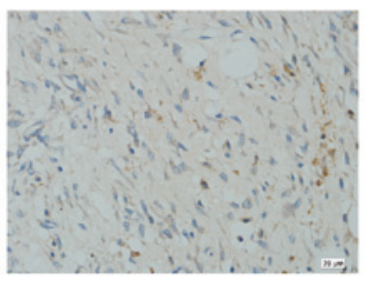

Hyperbaric oxygen

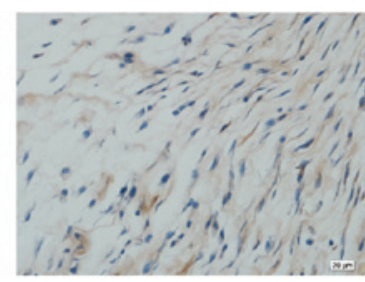

Control

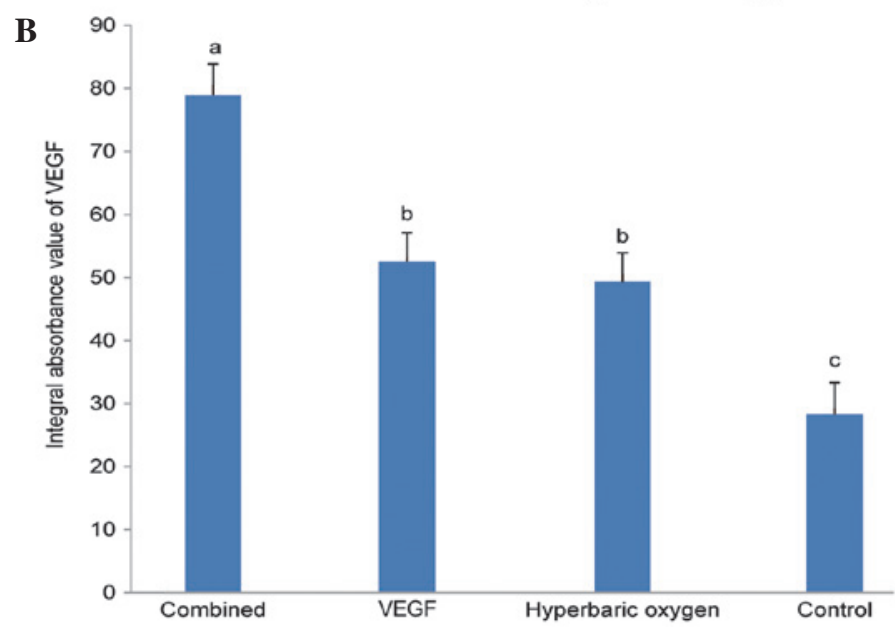

Figure 3. Comparison of VEGF expression between the four groups. (A) Representative fields of samples from the various groups under immunohistochemical staining (magnification, $\mathrm{x} 400$ ). (B) Histogram bars indicate the mean \pm standard deviation. Different letters indicate significant differences at the $\mathrm{P}<0.05$ level. VEGF, vascular endothelial growth factor.

VEGF release displayed an initial burst on the first day (25\%), followed by a sustained release of $\sim 10 \%$ from days $1-7$. On the seventh day, $\sim 92 \%$ of the VEGF had been released from the microspheres in total.

Gross observation and percentage of necrotic area. Seven days following treatment, the necrotic sections tended to fuse, scab and harden. The boundaries between necrotic and survival areas were stable. The necrotic area was black, rigid, and glabrous and it did not bleed when cut with a scalpel. The survival area was pink-white, tender, grew fine hair and it bled when cut (Fig. 1A).

The results of the necrotic area percentage are presented in Fig. 1B. The mean percentage of necrotic flap area in the control group $(49.66 \pm 2.64 \%)$ was significantly enlarged compared with that of the dual treatment group $(10.44 \pm 2.48 \%)$, the VEGF group $(26.85 \pm 1.77 \%)$ and the HBO group $(28.27 \pm 2.21 \%)$. No significant difference was detected between the VEGF group and the HBO group. The mean necrotic flap area percentage of the dual treatment group was significantly smaller than that of the VEGF group and the HBO group.

Histology. The morphological images of all groups on the seventh day following treatment is shown in Fig. 2A. The neovascularization results of intermediate area II for the four groups are illustrated in Fig. 2B. Consistent with the necrotic area percentage analysis, the neovascularization of the dual treatment group $\left(34.81 \pm 3.93 / \mathrm{mm}^{2}\right)$ was significantly higher than that of other groups. No significant difference was detected between the VEGF group $\left(22.96 \pm 3.29 / \mathrm{mm}^{2}\right)$ and the HBO group $\left(24.74 \pm 3.19 / \mathrm{mm}^{2}\right)$. In addition, the neovascular- ization of the controls $\left(16.68 \pm 2.69 / \mathrm{mm}^{2}\right)$ was lower than that of others.

Immunohistochemistry for VEGF. Seven days after the treatments, the results of immunohistochemical staining of the four groups were as follows (Fig. 3A). The IA values of VEGF of the dual treatment group, VEGF group, the HBO group and the controls were $78.97 \pm 4.90,52.54 \pm 4.55,49.32 \pm 4.62$ and $28.33 \pm 4.98$, respectively. Statistical analysis results corroborated the results of the histological analysis and necrotic area percentage analysis (Fig. 3B).

\section{Discussion}

In the present study, it was observed that VEGF or HBO administered after surgery improved the survival of random skin flaps. This is consistent with the results of previous studies $(2-4,17)$. There was no significant difference between the VEGF group and the HBO group. However, a combination therapy with VEGF and HBO led to improvement in the average survival compared with treatment with VEGF or HBO alone, suggesting that these agents act synergistically. For the combination group, the subcutaneous tissues had no obvious edema, congestion or neutrophil invasion. The number of novel blood vessels and the expression of VEGF of the combination group was significantly greater than that in the other groups. The control group treated with saline alone exhibited the highest percentage of necrotic area, the fewest number of novel blood vessels and the lowest VEGF expression.

A hypoxic environment has been shown to improve the VEGF expression (18-20). In damaged histiocytes, HBO 
stimulates the expression of hypoxia inducible factor 1 (HIF-1 $\alpha$ ), which binds to the VEGF transcription initiation site and promotes VEGF transcription and translation $(19,20)$. The upregulation of VEGF by HBO intervention may enhance its effect of promoting angiogenesis and vascularization. In addition, since the effect of VEGF administration is limited by the existing numbers of endothelial cells, increasing the number of endothelial cells may enhance its effects. A hypoxic environment has also been shown to promote the proliferation of endothelial cells (21). Thus, the enhanced effect of the combination of VEGF and HBO on the improvement of random skin flap survival may be explain the above described association of $\mathrm{HBO}$ with VEGF.

In conclusion, HBO or VEGF intervention can improve skin flap survival in rats. In addition, combination of VEGF and $\mathrm{HBO}$ did improve random skin flap survival to a greater extent than VEGF or HBO alone, suggesting that these two agents do potentiate one another. The results suggest that the combination of VEGF-loaded microspheres and hyperbaric oxygen may be a promising therapeutic intervention for the reduction of flap necrosis.

\section{Acknowledgements}

This study was supported by a grant from the Project of Science and Technology Bureau (no. HAS2012002).

\section{References}

1. Matsumura H, Yoshizawa N, Vedder NB and Watanabe K: Preconditioning of the distal portion of a rat random-pattern skin flap. Br J Plast Surg 54: 58-61, 2001.

2. Richards L, Lineaweaver WC, Stile F, Zhang J and Zhang F: Effect of hyperbaric oxygen therapy on the tubed pedicle flap survival in a rat model. Ann Plast Surg 50: 51-56, 2003.

3. Padubidri A and Browne E Jr: Effect of vascular endothelial growth factor (VEGF) on survival of random extension of axial pattern skin flaps in the rat. Ann Plast Surg 37: 604-611, 1996.

4. Kryger Z, Zhang F, Dogan T, Cheng C, Lineaweaver WC and Buncke HJ: The effects of VEGF on survival of a random flap in the rat: examination of various routes of administration. $\mathrm{Br}$ J Plast Surg 53: 234-239, 2000.

5. Khan A, Ashrafpour H, Huang N, et al: Acute local subcutaneous VEGF165 injection for augmentation of skin flap viability: efficacy and mechanism. Am J Physiol Regul Integr Comp Physiol 287: R1219-R1229, 2004.
6. Zhang T, Gong W, Li Z, et al: Efficacy of hyperbaric oxygen on survival of random pattern skin flap in diabetic rats. Undersea Hyperb Med 34: 335-339, 2007.

7. Ulkür E, Karagoz H, Ergun O, Celikoz B, Yildiz S and Yildirim S: The effect of hyperbaric oxygen therapy on the delay procedure. Plast Reconstr Surg 119: 86-94, 2007.

8. Kranke P, Bennett MH, Martyn-St James M, Schnabel A and Debus SE: Hyperbaric oxygen therapy for chronic wounds. Cochrane Database Syst Rev 4: CD004123, 2012.

9. Jung S, Wermker K, Poetschik H, Ziebura T and Kleinheinz J: The impact of hyperbaric oxygen therapy on serological values of vascular endothelial growth factor (VEGF) and basic fibroblast growth factor (bFGF). Head Face Med 6: 29, 2010.

10. Hunt TK and Pai MP: The effect of varying ambient oxygen tensions on wound metabolism and collagen synthesis. Surg Gynecol Obstet 135: 561-567, 1972.

11. Rinsch C, Quinodoz P, Pittet B, et al: Delivery of FGF-2 but not VEGF by encapsulated genetically engineered myoblasts improves survival and vascularization in a model of acute skin flap ischemia. Gene Ther 8: 523-533, 2001.

12. Mcfarlane RM, Deyoung G and Henry RA: The design of a pedicle flap in the rat to study necrosis and its prevention. Plast Reconstr Surg 35: 177-182, 1965.

13. Mandriota SJ, Pyke C, Di Sanza C, Quinodoz P, Pittet B and Pepper MS: Hypoxia-inducible angiopoietin-2 expression is mimicked by iodonium compounds and occurs in the rat brain and skin in response to systemic hypoxia and tissue ischemia. Am J Pathol 156: 2077-2089, 2000.

14. Ozkan O and Ozgentas HE: Combination of rat vest, teeth shortening and nail cutting to prevent autocannibalization and protect surgical flaps. Plast Reconstr Surg 117: 1671, 2006.

15. Chen Z, Wang T, Cai L, et al: Clinicopathological significance of non-small cell lung cancer with high prevalence of Oct-4 tumor cells. J Exp Clin Cancer Res 31: 10, 2012.

16. Shi ZR, Itzkowitz SH and Kim YS: A comparison of three immunoperoxidase techniques for antigen detection in colorectal carcinoma tissues. J Histochem Cytochem 36: 317-322, 1988.

17. da Rocha FP, Fagundes DJ, Pires JA and da Rocha FS: Effects of hyperbaric oxygen and $\mathrm{N}$-acetylcysteine in survival of random pattern skin flaps in rats. Indian J Plast Surg 45: 453-458, 2012

18. Daniel RA, Cardoso VK, Gois Jr E, et al: Effect of hyperbaric oxygen therapy on the intestinal ischemia reperfusion injury. Acta Cir Bras 26: 463-469, 2011.

19. Zhou Y, Liu XH, Qu SD, et al: Hyperbaric oxygen intervention on expression of hypoxia-inducible factor-1alpha and vascular endothelial growth factor in spinal cord injury models in rats. Chin Med J (Engl) 126: 3897-3903, 2013.

20. Ijichi H, Taketomi A, Yoshizumi T, et al: Hyperbaric oxygen induces vascular endothelial growth factor and reduces liver injury in regenerating rat liver after partial hepatectomy. J Hepatol 45: 28-34, 2006.

21. Ren P, Kang Z, Gu G, et al: Hyperbaric oxygen preconditioning promotes angiogenesis in rat liver after partial hepatectomy. Life Sci 83: 236-241, 2008. 